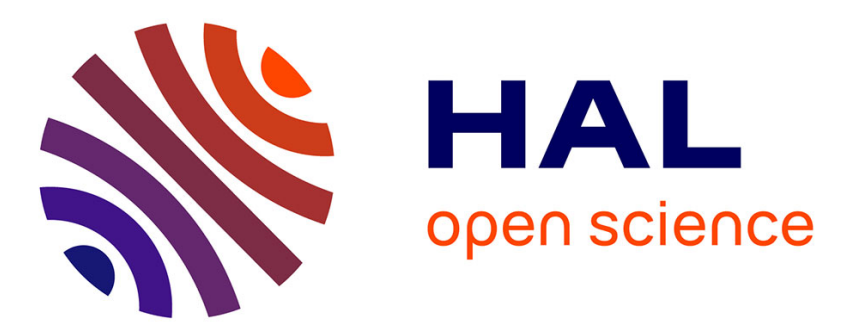

\title{
L'évolution de l'environnement numérique des musées. Des CD-Rom aux applications muséales et autres e-albums
}

Mathilde Miguet, Françoise Paquienséguy

\section{- To cite this version:}

Mathilde Miguet, Françoise Paquienséguy. L'évolution de l'environnement numérique des musées. Des CD-Rom aux applications muséales et autres e-albums. Interfaces numériques, 2019, 1-2, pp.79-92. 10.3166/LCN.13.1-2.69-93 . hal-02148413

\section{HAL Id: hal-02148413 \\ https://hal.science/hal-02148413}

Submitted on 12 Mar 2021

HAL is a multi-disciplinary open access archive for the deposit and dissemination of scientific research documents, whether they are published or not. The documents may come from teaching and research institutions in France or abroad, or from public or private research centers.
L'archive ouverte pluridisciplinaire HAL, est destinée au dépôt et à la diffusion de documents scientifiques de niveau recherche, publiés ou non, émanant des établissements d'enseignement et de recherche français ou étrangers, des laboratoires publics ou privés. 


\section{L'ÉVOLUTION DE L'ENVIRONNEMENT NUMÉRIQUE DES MUSÉES Des CD-Rom aux applications muséales et autres e-albums \\ MATHILDE MIGUET \\ FRANÇOISE PAQUIENSEGUY}

L'environnement numérique des musées a commencé avec les CD-Rom dans les années 1990 et n'a cessé de s'étoffer depuis. Pionniers et acteurs puissants, la Rmn-GP et le Centre Georges Pompidou ont développé depuis peu des e-albums et des applications smartphones à partir des grandes expositions que cet article analyse à deux niveaux. Premièrement, il montre comment les e-albums s'inscrivent dans la continuité des CDRom et en parallèle au catalogue d'exposition sur des logiques transmédia ; deuxièmement, il montre comment les e-albums et les applications smartphones sont des éléments désormais centraux de la stratégie de storytelling mise en place à très grande échelle par ces institutions culturelles à propos de grandes expositions, ou d'artistes incontournables (Monet, Rodin, Picasso, ...). Cette partie numérique de l'offre éditoriale des musées ouvre les portes d'une nouvelle médiation culturelle.

\section{Introduction}

Cet article s'intéresse aux ferments d'innovation de nouvelles productions et applications numériques productions, aujourd'hui incontournables ${ }^{1}$ des environnements numériques des institutions muséales, et tout particulièrement celles proposées par la Réunion des musées nationaux $(R m n)^{2}$ et le Centre Georges Pompidou, dénommées e-albums. Dans une logique différente du catalogue d'exposition, les e-albums sont pensés comme une synthèse informée et structurée d'une exposition dont ils permettent pour le moins de découvrir une sélection raisonnée des œuvres exposées, l'artiste qui les a créées, le contexte historique de référence sous la forme d'une application à télécharger afin de pouvoir I'utiliser sous IOS ou Android. Sans que ce produit éditorial devienne systématique pour autant, plusieurs expositions de rang international ont déjà donné lieu à des e-albums ou des applications mobiles $^{3}$, comme : Gauguin, Kupka, Picasso Mania, Clergue, Cartier, Niki de Saint Phalle, Velázquez, Hopper, ou encore Monet qui nous serviront d'exemple ici.

En effet, ces e-albums, centrés sur un artiste, s'inscrivent dans la lignée des CD-Rom culturels, objets d'un engouement sans pareil dans les années 1990 dont le plus célèbre

1. 495 Applications mobiles muséales et patrimoniales en France $(28 / 08 / 18)$ sur http://www.club-innovation-culture.fr/applications-mobiles-france-3/, consulté le 27/11/2018.

2. Devenue Réunion des Musées Nationaux-Grand Palais (Rmn-GP) en 2011.

3. https://www.grandpalais.fr/fr/les-applications-mobiles, consulté le 28/11/2018. 
aura été justement le fameux titre ${ }^{4}$ publié en 1995 par la Rmn, Le Louvre, peintures et palais ${ }^{5}$. Cependant, le succès du genre a été de courte durée, et les CD-Rom culturels ont progressivement disparu ${ }^{6}$ au profit d'autres formes multimédias et interactives proposées par les sites web à l'orée du siècle. C'est de ce double ancrage des e-albums dont il sera question ici, car ils sont à la fois les héritiers directs des CD-Rom culturels des années 1990 et partagent le quotidien des applications devenues familières. Cette dualité pèse d'ailleurs sur notre hypothèse centrale: malgré leurs liens incontestables avec les productions multimédias antérieures, les e-albums contemporains paraissent disruptifs car ils portent et intègrent des univers conceptuels et narratifs différents formant dispositif et non plus écosystème autonome. Par ailleurs, ces productions ${ }^{7}$ soulignent également les évolutions des institutions muséales tant sur le plan éditorial que sur celui de la médiation culturelle à partir et autour de la pièce maîtresse, l'exposition, incarnée par le catalogue d'exposition imprimé. En effet, nous référer aux fonctions principales du catalogue papier (Mayon, 2016) permet de voir dans quelle mesure les applications muséales et les ealbums les vérifient et les assument, car, comme le soulignent Muriel Amar et Agnès Vigué-Camus, chargées d'études à la Bibliothèque Publique d'Information du Centre Georges Pompidou «le néologisme e-album créé par les éditions Rmn-GP, introduit une distance $^{B}$ avec le catalogue imprimé qui brouille les signes d'identification d'un genre éditorial déjà complexe» (Amar, Vigué-Camus, 2017). Telles que définies par Mayon (2016), les fonctions premières et basiques du catalogue d'exposition et des applications et e-albums sont identiques (lister les œuvres, en montrer des reproductions et intégrer les notices textuelles). Cependant, l'e-album en sublime certaines grâce aux outils dont il est doté, le zoom et la définition des photos par exemple favorisent le lien avec l'art, et surtout d'autres fonctions caractéristiques méritent d'être étudiées comme faire ou laisser trace, favoriser la réception de l'exposition, accroître la notoriété de l'institution qui le produit faisant passer les e-albums et les applications de « documents sur l'exposition [à] documents de l'exposition » (Mayon, 2016).

\footnotetext{
4. Voir « Choisi pour vous, le cd-rom culture Le Louvre, peintures et palais, triomphe d'un musée imaginaire » Roger-Pierre Lagrange, Libération, 10 mars 1995, https://www. liberation.fr/ecrans/1995/03/10/choisi-pour-vous-cd-rom-culture-le-louvre-peinture-et-palaistriomphe-d-un-musee-imaginaire_128593, consulté le 12/11/2018.

5. Le Louvre, peintures et palais, Montparnasse Multimédia - Réunion des musées nationaux (1994).

6. https://www.latribune.fr/archives/2003/entreprises/id787f0df35096b402c1256e00003b36d9/lecd-rom-culturel-cherche-a-traverser-la-crise.html, consulté le 10/12/2018.

7. L'Ours dans la préhistoire, Fabrique cubiste, Fabrique romaine, Atelier Picasso, Pablo Picasso - 25 chefs d'œuvres expliqués aux enfants, Mooc Picasso, Artistes et Robot, et l'application du Grand Palais « tout en une ».

8. 1/support (application pour tablette vs livre imprimé) ; 2/rapport à l'exposition (sélectif vs exhaustif) ; $3 /$ durée de vie (éphémère vs atemporel) ; 4/prix (très bon marché vs très élevé) ; processus narratif spécifique à l'écriture numérique.
} 
Ainsi notre raisonnement se fera-t-il en deux temps. Nous montrerons d'abord en quoi les e-albums s'inscrivent dans la droite ligne des CD-Rom culturels produits à la fin des années 1990 afin d'introduire la filiation multimédia (Séguy, 1995) caractéristique intrinsèque des $C D$-Rom culturels, réincarnés dans les e-albums. Ensuite, nous cernerons comment cette inscription dans une lignée conceptuelle croisant art, numérique, interactivité et multimédia facilite un glissement du multimédia vers un transmédia contemporain (Jenkins, 2006), qui pense les e-albums au sein d'un dispositif communicationnel stratégique participant de l'environnement numérique des institutions muséales. Il change alors le statut de ces productions numériques. Nous proposerons donc une approche à la fois sémiologique et pragmatique pour dévoiler « I'univers narratif original » (Jenkins, 2006) des e-albums, à propos de la diffusion, de la narration, voire de la gamification.

Cet article s'appuie sur une partie du matériel produit par les auteurs dans le cadre d'un programme de recherche porté par Alexandra Saemmer : Catalogues d'exposition - Zone de tests ${ }^{9}$ et financé par le Labex Arts-H2H(2012-2015) mais aussi sur un corpus révisé et actualisé d'e-albums et d'applications pour smartphones et tablettes.

\section{En droite ligne des CD-Rom culturels}

Pour ceux et celles qui comme nous se sont intéressés aux produits multimédias interactifs produits à la fin des années 1990, les similitudes entre les CD-Rom culturels et les e-albums sautent aux yeux. En effet, les e-albums commercialisés depuis 2013 par la $R m n-G P$, principale éditrice française à ce jour, peuvent être considérés comme une version plus aboutie et plus maîtrisée des CD-Rom culturels dont ils tirent leçons, principes et règles. L'héritage qu'ils exploitent ainsi se dessine en creux car ces règles et principes n'ont jamais été clairement listés ou formalisés. En fait, la filiation n'est jamais revendiquée comme telle car une rupture générationnelle l'interdit : les concepteurs des CD-Rom venaient véritablement du secteur de celui du multimédia alors que les seconds sont généralement issus de celui de la communication numérique. En effet, entre les deux périodes (CD-Rom 1990-2003 et e-albums depuis 2013) se sont les productions web (sites, visite virtuelles, réseaux sociaux, web-documentaires, etc.), porteuses de I'environnement numérique le plus visible et permanent qui ont occupé ces professionnels

\subsection{Le multimédia première génération}

En 1996, une enquête sur les Premiers usages des CD-Rom des musées avait été commanditée par la Rmn auprès de trois chercheurs, Jean Davallon, Hana Gottesdiener et Joëlle Le Marec. Publiée en 2000 cette analyse expose clairement les freins rencontrés par les usagers, leurs attentes, leurs difficultés concrètes à l'usage et esquisse les écueils des

9. http://labex-arts-h2h.fr/catalogues-d-exposition-augmentes.html, consulté le 18 décembre 2018 
CD-Rom culturels, si nombreux dans les années 1990 que le genre n'y a pas survécu (Lavigne, 2010).

Les usagers de CD-Rom des musées formulaient alors plusieurs lourds griefs : 1/une procédure d'installation sur l'ordinateur à la fois interminable (à cause des lenteurs de la machine s'ajoutant aux temps de téléchargement) et complexe car obligeant à manier fichiers et procédures qui n'ont rien de transparents pour le néophyte, alors vite dépassé ; 2/ des lenteurs à l'exécution dues aux faibles capacités des processeurs de l'époque qui peinaient à ouvrir des images mal compressées ou des sons encore très lourds ; 3/ l'omniprésence de la voix-off, lassante pour l'usager car elle se déclenche automatiquement, ne peut être supprimée et gêne lorsqu'elle s'associe à un texte long que l'écran incite à lire ; 4/ une désorientation fréquente dans le produit à cause d'architectures trop complexes, accrue par l'absence d'un moyen aisé de rebrousser chemin puisque le back du navigateur n'existe pas encore, et pour cause : le navigateur non plus! Découvrant arborescence et hypertextualité, les usagers de l'époque ne parviennent pas à se créer une représentation mentale de la structure du produit et sont soit perdus, soit incapables de maîtriser leurs parcours à tel point que la plupart d'entre eux déclarent tout interrompre et repartir du début afin de mieux se repérer dans le produit interactif multimédia, quand l'arrêt n'est pas définitif. Bref, ils n'ont pu développer encore aucune littératie numérique dont l'appropriation du micro-ordinateur formalisera d'ailleurs la première étape (Jouët, 1987).

Presque dans le même temps, en 1999, nous tentions une première définition scientifique du terme « multimédia » très professionnel et à la mode ; au-delà des formes très variées sous lesquelles il commençait à être présent (CD-Rom éducatifs, culturels, ludiques, ludoéducatifs, premiers sites avec des interfaces graphiques et du son...). Ainsi, «le multimédia reposait sur l'exploitation des possibilités techniques du moment générant une démultiplication des vecteurs de diffusion de l'information ; cette multiplicité étant mise à la disposition du concepteur de l'application pour exprimer ses idées, choix ou ligne éditoriale. » (Séguy, 1999). Plusieurs registres étaient déjà présents pour le caractériser en cette toute fin de $X X^{\mathrm{e}}$ siècle. D'abord le son qui se matérialisait déjà de bien des façons : les bruitages, les sons de confirmation, les voix-off et/ou sous-titrages, le choix de la langue, la musique d'accompagnement ou la création d'une ambiance sonore. Ensuite, le visuel qui, dépassant le seul registre textuel, prenait des formes souvent innovantes : les icônes, les vignettes, les boutons, les fonds d'écran, les pop-up, les éléments graphiques, l'image fixe, les effets visuels. Et enfin l'animation même de la page écran : la vidéo encore saccadée, le diaporama, le panoramique et autres effets cinématiques.

Inutile de faire une démonstration détaillée assurant que les e-albums intègrent la plupart de ces possibilités, et que les freins liés à la lenteur, l'inconnu ou la complexité ont été levés : nous le vérifions déjà au quotidien avec les interfaces que nous manipulons. Disons simplement que, depuis 1997, les freins technologiques ont tous été levés et 
soulignons les progrès de l'usager (Paquienséguy, 2018) qui maîtrise maintenant les manipulations et fonctionnalités nécessaires à leur utilisation, du moins pour celui qui correspond à la cible visée par les e-albums. Aujourd'hui, en quelques minutes, grâce à une connexion 4G sur ITunes, et d'un doigt, l'e-album Monet pourtant lourd de 634 Mo, s'installe sur la tablette ou le smartphone. Puis, le doigt glisse, tourne la page, certes de façon virtuelle mais avec une aisance assurée (Saemmer, 2015) pour pincer-glisser, pincer-ouvrir, toucher-glisser, toucher-tourner, etc. De plus, la structuration de ces produits est facilitée par des outils et fonctionnalités qui rendent la manipulation sinon intuitive, ou user friendly comme les discours promotionnels l'annoncent, du moins claire et compréhensible. Par exemple, la navigation dans les pages du e-album se fait en un clic digital, grâce à ce qui s'appelait dans les années 1995 un menu d'hypernavigation (Séguy, 1999) devenu le plan du site dans les sites web actuels et que nous retrouvons dans Hopper sous la forme d'une carte, sous celle d'un simple sommaire dans Monet, d'un story-board dans Gauguin I'Alchimiste ou d'un slogan publicitaire obsédant ${ }^{10}$ dans Niki de St Phalle. Cette représentation visuelle de l'ensemble du produit, facilite la compréhension de sa structuration et permet également à l'utilisateur de visualiser son parcours. En effet, les pages déjà ouvertes se grisent au fur et à mesure de la consultation alors que la page en cours de consultation marque le traditionnel « vous êtes ici » d'une façon ou d'une autre (encadré, grisé, couleur rouge, etc.).

D'ailleurs dès 2000, nous affirmions que ces menus d'hypernavigation constituaient, et constitueraient, un des éléments fondamentaux de l'inter-activité de structure des produits multimédias. Cette fonctionnalité est d'ailleurs aujourd'hui magnifiée par les interfaces de Windows 8 ou des smartphones qui affichent les vignettes ou icônes de toutes les fonctions ou applications disponibles. De plus, d'autres outils présents à l'écran ont également progres-sivement codifié l'interaction jusqu'à former des normes de fait quant à la structure d'une interface, tels les liens constants présents sur toutes les pages afin de retrouver la page d'accueil, d'imprimer, de se débarrasser de la voix-off ou de la musique, de partager ou d'archiver l'écran, sans parler des flèches suivant/précédent, ou de la réglette précédemment dite « réglette magnétoscope » associée à toute vidéo et des liens sociaux omniprésents. Fonctionnalités de base de la manipulation des produits multimédias, ces éléments ont sans doute formé le premier socle de la littératie numérique des utilisateurs.

Pour les productions éditoriales muséales de l'époque, le maître mot est interactivité (Le Marec, 2004). Deux éléments sont à en retenir. D'abord, c'est bien elle, qui, par essence puisqu'elle porte le choix, ouvre les portes des catalogues comme des réserves et libère à la fois le visiteur virtuel et le conservateur des contraintes de l'espace d'une part et de la

10. « Je m'appelle Niki de St Phalle, et je fais des œuvres monumentales ». 
linéarité des parcours qu'il impose, de l'autre. D'ailleurs la première forme la plus investie par les musées sera celle de la visite virtuelle, le Louvre se posant alors en pionner.

\subsection{L'évolution du multimédia, de ses concepteurs et de ses usagers}

Comme d'autres catégories de produits, ludo-éducatifs par exemple, les e-albums de la $R m n-G P$ ont appris des pionniers et ce d'autant plus que les concepteurs d'aujourd'hui, installés à des postes clefs des services d'édition numérique de grandes institutions culturelles ont fait leurs études dans le multimédia et l'interactivité justement à cette période si particulière.

En effet, la décennie 1990/2000 a expérimenté des formes, des outils, des formats, des procédés narratifs et se caractérise par une forte créativité. Elle a correspondu à un temps d'émergence foisonnant dont certains syntagmes résonnent encore à nos oreilles : «le premier roman interactif ${ }^{11}$; « les générateurs d'écriture » 12 ; la visite virtuelle $\left(360^{\circ}\right.$ ou 3D) que le site web du Louvre sera le premier à proposer dès 1998 et à prolonger l'expérience jusqu'à aujourd'hui ${ }^{13}$, nous l'avons déjà souligné.

A priori les concepteurs, producteurs et éditeurs d'aujourd'hui, dont l'âge fait du web la seule référence en la matière, savent ce qu'il ne faut pas reproduire. Ils ont appris des retours utilisateurs (avis, notes, commentaires, forum utilisateurs...) et maîtrisent désormais les principaux codes et règles d'écriture et de composition d'interfaces, à tel point que les e-albums contemporains remplacent les CD-Rom culturels d'hier dont ils ont levé les freins à l'usage les plus puissants. Nous pourrions nous appuyer sur deux exemples qui illustrent cette transition. Formé au multimédia sur CD-Rom Thomas Bijon pilote aujourd'hui le Département audiovisuel et multimédia de la $R m n-G P$ qui profite ainsi de son expérience en la matière, décalée des logiques print/web ou communication digitale actuelles. La Rmn-GP souligne d'ailleurs elle-même ses recherches éditoriales ${ }^{14}$ "nous n'avons cessé de proposer de nouvelles publications numériques et d'expérimenter: d'autres formats (les catalogues Redon, Stein et Cézanne), d'autres formes d'écriture (Hopper), d'autres modes de narration (Cartier), un métissage entre livre et documentaire (Depardon), la possibilité d'une collection $100 \%$ numérique ("Les plus beaux... de l'histoire de l'art ») et bien sûr de nouvelles possibilités graphiques avec toute une ergonomie à réinventer (Niki de Saint Phalle, Durand-Ruel, Velázquez)».

11. Intitulé Orange, il a été présenté à l'exposition Les Immatériaux au Centre G. Pompidou en 1985, https://www.centrepompidou.fr/cpv/resource/cRyd8q/r6rM4jx, consulté le 17/11/2018.

12. Dispositif inventé par Jean-Pierre Balpe, voir http://entrelapageetlecran.nt2.ca/ auteur/jeanpierre-balpe, consulté le 17/11/2018.

13. Le Musée du Louvre propose des visites virtuelles des salles grâce au soutien de la Fondation Shiseido; celui d'Orsay celles d'expositions passées grâce à la Fondation espagnole Mapfre; la visite virtuelle est devenue un élément incontournable de l'environnement numérique des institutions muséales (http://www.club-innovation-culture.fr/visites-virtuellesdes-musees-et-des-lieux-culturels/\#ancre3), consulté le 20/12/2018.

14. https://www.grandpalais.fr/ru/node/15558, consulté le 26/11/2018. 
Différemment, mais en complémentarité, Paul Mourey, Head of Digital du Centre Georges Pompidou s'est formé au marketing digital et travaille à la convergence des supports (smartphones et tablettes) et des productions (applications, $C D$, réseaux sociaux, sites web dédiés, etc.) pour une meilleure structuration de l'environnement numérique du Centre, ce qu'il résume ainsi «je pilote la stratégie numérique transverse de I'institution ».

Quant au Musée d'Orsay, il a créé une Direction du Numérique en mars 2018, laquelle a intégré l'ancien service Internet \& Multimédia afin de mettre en place une stratégie plus globale axée sur la convergence des médias, autrement dit le transmédia.

Ce mouvement chez des concepteurs et producteurs (Collet, 2018) est accompagné par une transformation significative de la cible. En effet, les consommateurs de e-albums se sont progressivement forgés une littératie numérique vérifiée dans la population potentiellement concernée puisque $18 \%$ des Français profitent pleinement de leur smartphone pour leurs loisirs et que $55 \%$ des Français téléchargent des applications régulièrement (Baromètre du Numérique, Arcep) ${ }^{15}$. Autrement dit, le moment est peutêtre propice au succès des e-albums et autres applications d'art car en presque 20 ans (1996-2014), c'est surtout l'usager des produits interactifs multimédias qui a développé de nombreuses compétences grâce à l'explosion de son équipement, à ses usages diversifiés d'Internet et à son aisance à manipuler des écrans tactiles. Pour les deux tiers des personnes ayant accès à Internet, ces applications les ont rendues habiles en téléchargement, archivage, consultation, navigation, en écran, en réseau et les ont conduites à se saisir de différents contrats de lecture ${ }^{16}$ en présence (hypertextes, paratextes, textes courts, boutons sémantiques, règles alphabétiques, cartes, sous-titrage vidéo, etc.). Ces consommateurs, usagers d'applications entre autres muséales ou culturelles ont développés des « arts de faire» (De Certeau, 1980). De la même façon, jusqu'à 40 ans, ils cernent mieux l'offre ${ }^{17}$ et ne s'étonnent plus de produits nouveaux, hybrides, dérivés, multi ou transmédia. Si l'on ne peut précisément parler de | reconnaissance d'un_genre en la matière (Odin, 2000) les approches de type sémio-

\footnotetext{
15. $69 \%$ des Français s'estiment compétents pour utiliser un smartphone ; $67 \%$ pour utiliser un ordinateur (+ 12 points) ; $62 \%$ pour utiliser une tablette. Baromètre du numérique novembre 2017. ARCEP : https://www.arcep.fr/uploads/tx_gspublication/ barometre_du_numerique-2017-271117.pdf, consulté le 27/10/2018.

16. Bélisle Cl., Ducharme Ch. (2003). Contrats de lecture. Bulletin des bibliothèques de France (BBF), $n^{\circ} 3$, p. 74-86.

17. $\quad$ https://www.arcep.fr/uploads/tx_gspublication/barometre_du_numerique-2017presentation-conf-271117.pdf, consulté le 28 octobre 2018.
} 
pragmatiques $^{18}$ que nous sollicitons, permettent de penser et de suivre ce double mouvement qui fait sens pour les concepteurs comme pour les usagers (Jahjah, 2014).

De façon caractéristique, le multimédia permet de diversifier les vecteurs d'information soit en cherchant la déclinaison de mêmes contenus informationnels ou narratifs sur plusieurs natures de médias (image, texte, son, animation, 3D, etc.), soit en cherchant la complémentarité entre les médias utilisés (print, web, vidéos, applications etc.), offrant alors une richesse et une valeur ajoutée supplémentaires venues d'un environnement numérique complet. Nous serions donc volontiers tentées de dire que le multimédia se vérifie à l'intérieur de bien des produits issus des industries culturelles d'abord car il cumule ou propose différents vecteurs de transmission, la façon dont il exploite les relations entre le texte et l'image (Laborderie, Juhel, 2016) par exemple en témoigne ; ensuite, parce qu'il est magnifié par la chronologie de publication ou de diffusion de ces productions diversifiées au sein de l'environnement numérique muséal. Celui-ci nous conduit à convoquer la notion de transmédia storytelling proposé par Henry Jenkins en 2003 dans un article paru dans Technological Review ${ }^{19}$, et stabilisée en 2006 dans son livre Convergence culture (Jenkins, 2006) pour considérer le transmédia comme la forme contemporaine du multimédia sur la base des deux dimensions des stratégies transmédia à l'œuvre, l'une liée à la diffusion, l'autre à la narration. D'ailleurs, par choix, nous laisserons de côté la question des supports et des formats pour nous concentrer sur I'univers narratif.

\section{Du multimédia au transmédia, et au storytelling}

\subsection{Du multimédia au transmédia}

Le multimédia par son cumul des vecteurs de diffusion porte en germes le transmédia, du moins le pensons-nous. En effet, le poids et l'omniprésence des interfaces ont permis l'absorption de l'interactivité par le multimédia, et encore dans le cas des interfaces tactiles où le doigt règne en maître (Durand, 2009). Mettant ainsi les registres formels de l'interface au premier plan (découpage de l'écran, animations, menus déroulants, titres, etc.) le multimédia constitue un tout, un univers de ressources et un potentiel de chemins pour les explorer (Lavigne, 2007) qui porte déjà le double principe d'une forme ouverte de chronologie et de redondance ou de point similitudes des informations diffusées. En tant qu'œuvre multimédia, le e-album Hopper par exemple est particulièrement connu pour sa carte qui expose dans un seul et même écran les parcours possibles, les éléments déjà visionnés en les mettant tous au même niveau puisqu'ils s'ouvrent tous d'un seul clic.

18. Marc Jahjah fait référence ici aux travaux de Vincent Liquète (2010), Joëlle Le Marec (2003). Il présentait une notion qualifiée «d'autorité distribuée », lors du colloque Écridil Montréal 30 avril- $1^{\text {er }}$ mai 2018 : http://ecridil.ex-situ.info/ programme, consulté le 20/08/2018. 19. Transmedia storytelling - Moving characters from books to films to video games can make them stronger and more compelling »: https://www.technologyreview.com/ s/401760/ transmédia-storytelling/, consulté le 12/12/2018. 


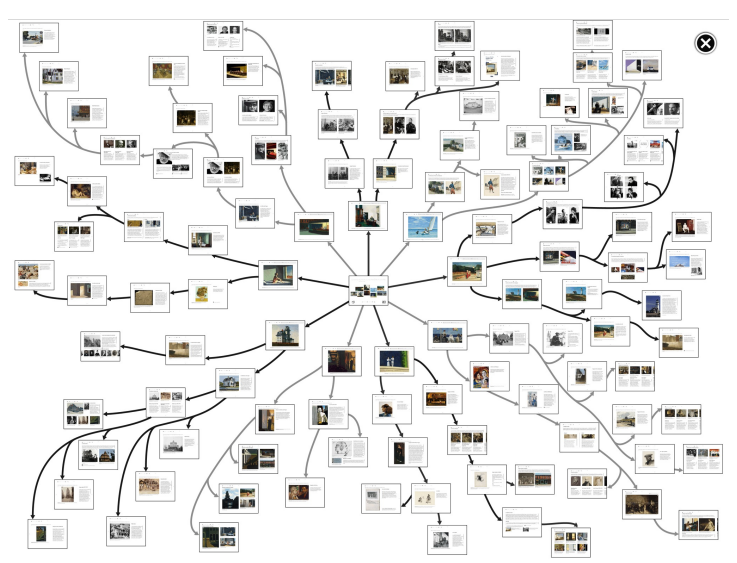

Map de l'e-album Edward Hopper d'une fenêtre à l'autre. (c) RMN-Grand Palais

Ainsi, comme dans le transmédia, des informations convergentes d'une part et redondantes de l'autre (le logo de l'institution, l'œuvre emblématique de l'exposition, la courte présentation ou la phrase générique par exemple) font être à disposition dans la production multimédia. Jusqu'aux années 2010 (Le Diberder, 2006), cette production prend le plus souvent la forme d'un site web puisque c'est bien cette forme qui a supplanté les CD-Rom culturels, et principalement ceux qui se voulaient encyclopédiques à propos d'un artiste ou d'une institution muséale (Schafer et Thierry, 2011). On pense par exemple à ceux présentant Rodin, Versailles, Cézanne ou encore Van Gogh (Lavigne, 2007, p. 17). Le cas d'Hopper est d'ailleurs ici très intéressant à reprendre puisque le ealbum propose fréquemment des liens qui sortent complètement le lecteur de son propre univers pour le propulser, par exemple, dans celui de Gallica ou de I'Ina où des archives lui sont proposées, tout comme le ferait un site web. Les CD-Rom multimédias, puis les sites web qui s'en sont inspirés représentent donc un univers de ressources et de possibles sur lequel le transmédia pourra s'appuyer en les exploitant selon une logique éditoriale (celle de la segmentation et de la diffusion concomitante) qui le caractérise.

\subsection{Transmédia et stratégies de diffusion}

Revenons d'abord sur la définition du transmédia storytelling par Jenkins pour qui il représente " un processus à travers lequel les éléments d'une fiction sont dispersés sur plusieurs plateformes médiatiques dans le but de créer une expérience de divertissement coordonnée et unifiée ». Si le parallèle se fait aisément entre multimédia et transmédia puisque le premier fait reposer la construction du sens sur des vecteurs différents comme nous l'avons montré plus haut, l'expérience de divertissement reste à construire dans le registre de la stratégie de diffusion pour les institutions culturelles qui éditent les ealbums étudiés. En effet, l'étude du dispositif transmédia déployé autour des grandes expositions est un exemple des stratégies de diffusion de la $R m n-G P$ qui structure et coordonne ainsi l'expérience utilisateur, qu'il soit visiteur, ou pas, de l'exposition au Grand 
Palais. À ce jour de décembre 2018, ce sont soixante-deux applications ${ }^{20}$ liées à des expositions de la Rmn-GP qui sont disponibles sur l'Appstore, chiffre en perpétuelle hausse puisque les récents réaménagements du Nouveau Grand Palais lui permettent d'accueillir trois grandes expositions en même temps. La majorité des e-albums intègrent des stratégies récurrentes et bien rodées qui valident un processus industrialisé qui vise à la fois la rareté (l'exposition ou sa visite avec le commissaire d'exposition) la masse (le site web) ; les amateurs éclairés (cours d'histoire de l'art en lien avec l'exposition), les novices (visite découverte qui se fixent sur quelques œuvres seulement), la peinture (ealbum, catalogue) et des entrées complémentaires (projection de documentaires, ateliers), etc.

Si nous avons choisi le e-album Monet pour l'illustrer, c'est d'abord qu'il est un des premiers à intégrer cette stratégie et qu'il est également le premier à bénéficier d'une mise à jour afin de faire perdurer le récit, sans cesse réinventé. Il vise alors la permanence latente (ou virtuelle) de l'exposition. En effet, l'exposition centrale Claude Monet 1840-1926, s'est tenue aux Galeries nationales du Grand Palais du 22 septembre 2010 au 24 janvier 2011 et c'est cette exposition qui a donné lieu au e-album. Révisé et actualisé en 2015, il a été remis dans l'actualité, à chaque grande exposition Monet en région ou à Giverny, et surtout en prévision de la nouvelle exposition parisienne à I'Orangerie en 2018, «Nymphéas, l'abstraction américaine et le dernier Monet »21. Le storytelling s'étale ici sur presque 10 ans. En effet, après l'ouverture de l'exposition le 22 septembre et la publication du catalogue la même semaine, un teaser de 9'34" est disponible sur YouTube le 14 novembre. Comme toujours désormais, l'e-album, n'est mis en vente via téléchargement que le 8 avril 2011, soit plus de deux mois après la fermeture de l'exposition qui remporte un franc succès (plus de 900000 visiteurs) et s'achève par un marathon alors inédit de quatre jours trois nuits d'ouverture non-stop. Il connaîtra une belle destinée puisque le e-album sera la $7^{\mathrm{e}}$ application la plus téléchargée, toutes catégories confondues. Le 20 octobre 2011 l'application est mise en ligne et devient gratuite. Elle gagne alors de nombreux prix : Double Webby Awards winner, FWA Site of the Month, Strategies special prize 2011 et Eurobest 2011 Grand Prix Interactive ${ }^{22}$. En juillet 2015, c'est une version révisée et enrichie qui est à nouveau disponible à la vente, en parallèle de la première version gratuite toujours accessible en ligne jusqu'en juillet 2018. Pendant l'ouverture de l'exposition, I'offre des activités de médiation est très conséquente, comme toujours aux Galeries du Grand Palais ${ }^{23}$. Autrement dit, la présence médiatique et discursive est continue sur le principe du relai, caractéristique de la

\footnotetext{
20. https://www.grandpalais.fr/apps/mobile/appipad.html, consulté le 18 décembre 2018.

21. 13 avril-20 août 2018.

22. www.monet2010.com, consulté le 13/12/2018.

23. On peut s'en faire une idée précise à propos de la période et des expositions en cours : https://www.grandpalais.fr/pdf/Offre_Mediation_GrandPalais_Automne2018.pdf
} 
stratégie de diffusion/médiation de la Rmn-GP. La stratégie de diffusion du e-album Monet l'inscrit dans un dispositif qui s'étend au-delà du Grand Palais et résonne avec des productions indépendantes, issue de Giverny ou de musées étrangers (Met New-York) ou provinciaux (Bordeaux). En tant que produit multimédia le e-album, même si on est sensible à son esthétique, ne propose aucun outil ou fonctionnalité qui en ferait l'originalité ou la spécificité : c'est son insertion au sein d'un dispositif d'une ampleur inégalée, tout comme l'exposition qui le spécifie. Se retrouve ici vérifiée ou plutôt transposée une des fonctions centrales du catalogue d'exposition déclinées par Mayon (2016) : « Le catalogue d'exposition [...] comme un moyen de diffusion, de publicité pour l'exposition». Certes, le e-album Monet est subordonné à l'exposition de 2010 dans les galeries du Grand Palais, mais il devient ensuite, à la fin de celle-ci, sous sa forme web, l'avatar de l'exposition qu'il fait vivre, réincarne, comme la visite virtuelle de l'exposition Impressionnistes et les Post-Impressionistes ${ }^{24}$ à Orsay par exemple. Autrement dit, le ealbum assure une permanence, une rémanence de l'exposition que les stratégies de storytelling entretiennent.

Le e-album Hopper montre également une stratégie transmédia de storytelling riche et complète qui a donné lieu à un dispositif conséquent que nous retraçons à grands traits $^{25}$ : I'exposition parisienne court du 10 octobre 2012 au 28 janvier 2013, mais quatre produits anticipent l'ouverture de l'exposition. Le livre Hopper Ombre et Lumière du commissaire d'exposition Didier Ottinger, publié le 20 septembre, suivi par le catalogue de l'exposition disponible à partir du 28 septembre, complété le $1^{\text {er }}$ octobre par la version anglaise du livre et un album papier de l'exposition lui-même disponible le 6 octobre. Sorties qui donnent lieu à des conférences et communiqués de presse. Pendant l'exposition des activités et productions de nature très différentes viennent se superposer à ce premier lot : une visite thématique de Paris en relation avec Hopper en novembre, un photoreportage sur le montage de l'exposition, assuré par Ferrante Ferranti, mis en ligne $^{26}$ en novembre 2012, un livre audio qui parait le 27 décembre et enfin deux séances de projection autour d'Hopper en janvier 2013, dont une pour clore l'exposition. Cependant, au vu de son succès, celle-ci sera prolongée du $1^{\text {er }}$ au 3 février sur une formule non-stop inauguré par le Grand-Palais avec Monet. Environ dix jours après la fermeture définitive de l'exposition, le 12 février 2013, paraît l'e-album conçu par Didier Ottinger Hopper d'une fenêtre à l'autre, qui permet de la revoir ou de la découvrir. En dernier lieu, une activité participative créative est proposée sur le site de l'exposition $M a$

\footnotetext{
24. http://exposiciones.fundacionmapfre.org/impresionistasypostimpresionistas/visita_virtual/vis ita_virtual.html, consulté le 27 novembre 2018.

25. Paquienséguy F. (2018). Multimédias interactifs, les progrès de l'usager . Théorème, $\mathrm{n}^{\circ} 29$, p. $97-108$.

26. Toujours disponible sur https://www.grandpalais.fr/fr/article/montage-de-lexpo-hopper, consulté le 13/10/2018.
} 
$B D$ Hopper invitant les internautes à inventer une bande dessinée autour et à partir de l'univers pictural du peintre jusqu'au 28 mars 2014.

La stratégie Hopper fonctionne dans un écosystème riche mais autarcique, totalement dépendant de la question des droits de reproduction ${ }^{27}$, de la notoriété internationale de l'artiste, de l'absence de partenaire muséal en Europe, elle n'est pas portée dans la durée. Comme plusieurs CD-rom avant, Hopper, d'une fenêtre à l'autre, laissera sa trace grâce à un des outils qu'il proposait la carte intuitive, menu d'hypernavigation réinventé. D'ailleurs, il s'inscrivait dans une démarche de recherche-action financée par le CNL et n'abonde pas vraiment aux collections numériques de la $R m n-G P$. Nous notons cependant que sa particularité vérifie une des fonctions centrales du catalogue (Mayon, 2016) : la justification du propos de l'exposition. Didier Ottinger est considéré comme LE spécialiste d'Hopper et cet e-album, dont il est le concepteur unique, le valorise fortement, d'autant plus que son ouvrage sur Hopper sort en parallèle. Ainsi le e-album, comme le catalogue antérieurement, met « en avant le commissaire d'exposition dès la couverture [...] De médiateur entre l'artiste, les ceuvres et le public, le catalogue devient alors médiateur entre l'institution et son public. » (Mayon, 2016). Il en va d'ailleurs de même pour l'ealbum Gauguin L'Alchimiste de Stéphane Guégan.

Les stratégies de diffusion de productions numériques des institutions muséales sont à la fois riches et nombreuses, de plus en plus pensées de façon transversale et témoignent d'une véritable structuration ou construction de leur environnement numérique. Pour illustration, la nouvelle stratégie intégratrice du Grand Palais qui a lancé le 21 septembre dernier "sa nouvelle application mobile "tout en une "qui permet de suivre son actualité, préparer sa venue, vivre pleinement les expositions et les événements, et conserver en souvenir ses cuvvres préférées, ses plus belles photographies et ses meilleurs moments de visite ${ }^{28}$. » Tout un environnement numérique embarqué !

\subsection{Transmédia et storytelling}

Si on s'en tient à l'acception de Jenkins, ce qui est nouveau au sujet des e-albums serait leur caractéristique qualifiée de transmédia storytelling (au sens de Jenkins (2006): relevant d'un « univers narratif original»). Mais il n'en semble rien : cette apparence réside dans une multiplicité variable des supports utilisés. En effet, ces apports techniques, qui se traduisent de façon tactile ou dans la navigation, n'offrent pas pour autant de nouveauté en termes de narration, et il n'y a pas de réel récit en propre par ailleurs... et c'est là que le bât blesse.

Pour cela, il faut revenir sur ce que représente le transmédia. Si les e-albums déclinent techniques (zoom, 3D, sous-titrages, etc.) et formes (audiovisuelles, sonores, etc.) de ce

27. Interview Thomas Bijon par Françoise Paquienséguy.

28. http://www.club-innovation-culture.fr/grand-palais-nouvelle-application-mobile/, consulté le $18 / 12 / 2018$ 
qui constitue le transmédia; il n'en reste pas moins qu'on ne peut les rattacher systématiquement à la notion de transmédia de Jenkins. En effet, pour cet auteur, I'univers imaginaire que développe l'expérience transmédia n'apparaît pas comme celui d'une œuvre achevée, mais celui d'une œuvre en réel ou en apparent développement, activité, ou action qui constitue « le récit, la narration d'un univers ». Pour en prendre un exemple, mais en se tenant loin du point de vue de l'historien de l'art, nous dirions qu'un peintre comme Hopper n'a pas pensé un univers en créant ses œuvres, si ce n'est celui construit a posteriori et pensé chronologiquement par la vision de Didier Ottinger à partir de l'œuvre d'Hopper présentée comme une « généalogie du réalisme américain » ${ }^{29}$. Certes, Hopper a développé une peinture avec sa grammaire et son symbolisme. Une peinture indéniablement empreinte de tension, de dramaturgie pour chaque tableau, qui puise sa source d'inspiration dans une Amérique dont il livre le récit pour certaines représentations, mais qui ne fait pas récit global a priori. La cohérence est de thématique et d'expression, elle n'est pas de narration dans son ensemble. Cette caractéristique s'applique à la majorité des catalogues d'œuvres, même si l'e-album Hopper se voulait un catalogue raisonné, il apparaît à la fois comme un tout (une sélection de neuf tableaux) et comme un index (chaque tableau faisant rubrique, riche, instruite et s'éloignant du peintre et du tableau pour retrouver le contexte et l'environnement qui lui était contemporain).

En ce sens, l'e-album et le catalogue d'exposition partagent la même fonction de médiation qui concourt à la réception de l'exposition; ils développent tous les deux le propos et un approfondissement de la connaissance des œuvres, de l'artiste et de sa période. Ici, Didier Ottinger raconte, en tant que commissaire d'exposition et passeur, en tant que spécialiste, metteur en scène et médiateur. Il fait découvrir SA vision d'un artiste à un public français, qui n'avait jamais pu voir ces peintures et gravures avant l'exposition parisienne. Oui, le médiateur raconte, car un storyteller est avant tout un conteur de I'histoire et de l'œuvre d'Hopper de multiples façons et sur des registres aussi différents. D'ailleurs, certains, élaborés durant l'exposition pouvaient même paraître irrespectueux, telle la $B D$ Hopper $^{30}$ qui donnait le verbe à la peinture... De fait, ce sont les applications jeunesse ou de vulgarisation, dites de divertissement, qui seules et classiquement tendaient vers une forme de gamification (L'Ours dans la préhistoire; Fabrique cubiste; Fabrique romaine, l'Atelier Picasso; Pablo Picasso, 25 chefs d'œuvre expliqués aux enfants : I'application; ...).

Or, une des vertus du transmédia, via des fonctionnalités de développement imaginaire et ludique, réside bien dans le fait de permettre une appropriation par l'utilisateur d'un

29. Interview de Didier Ottinger, dans l'émission de Pascale Clark Comme on nous parle, du 9 octobre 2012 : http://www.franceinter.fr/dossier-edward-hopper, consulté le 13/10/2018.

30 https://www.grandpalais.fr/fr/article/joue-ma-bd-hopper, consulté le 12/12/2018. 
imaginaire ouvert, auquel il va pouvoir participer, y compris en co-construisant l'application. Si le monde d'Hopper demeure déjà fermé, l'univers proposé reste figé, de façon définitive sans doute, car Hopper, comme la plupart des autres artistes sujets de ealbum, n'est plus là pour apporter de nouveaux tableaux, de nouveaux indices, qui permettraient selon les réactions et interactions du public de mener un développement ludique, collectif et parallèle de/à l'œuvre, tels que Jenkins les suggère. C'est donc à un autre niveau qu'il faut jouer, celui de l'utilisateur, du visiteur, et de sa propre expérience, toujours possiblement évolutive au contact de l'environnement numérique proposé par I'institution muséale. L'application Tout en une ${ }^{31}$ proposée le 21 septembre 2018 se met à jour à chaque visite au Grand Palais et s'enrichit en permanence de photos, historiques et téléchargements sur site à chaque visite d'une exposition. De fait, pour comprendre ce qu'amorce, peut-être, cette application, il nous faut évoquer deux raisons supplémentaires qui empêchaient la réussite des tentatives d'approcher au plus près le transmédia storytelling dans ses vertus de développement d'univers et de co-construction par I'utilisateur: jusqu'alors la gamification absente ou insuffisante et le cadre d'un développement économique contraint.

En effet, le développement ludique ou gamification s'entend ici comme l'ensemble des techniques d'incitation à la participation héritées des jeux vidéo et s'inspire des mécanismes ludoéducatifs (Jahjah, 2014). Les mécaniques ${ }^{32}$, les dynamiques du gamedesign reposent sur les enjeux destinés au joueur: gagner des points et des récompenses, collectionner des objets, résoudre une ou plusieurs énigmes étape après étape pour mener à bien sa quête ${ }^{33}$, explorer et maîtriser un univers, faire vivre son univers en se connectant régulièrement et fréquemment, accéder à une personnalisation du service, acquérir un statut dans le jeu (intégrer un club), contribuer à la collectivité, échanger entre joueurs. De là, les apports de la gamification en lien avec les univers transmédiatiques ne semblaient pas avoir prise sur les e-albums. De la même façon, alors que dans les années 1990-2000, les concepteurs faisaient la promotion d'une nouvelle approche dite ludo-pédagogique et interactive développée par les CD-Rom culturels (Lavigne, 2007). Nous voyions apparaître la réitération d'un discours porté par l'institution muséale toujours aussi techniciste: les potentialités de l'outil ou de l'application présupposant les pratiques effectives ; « le fantasme du visiteur créatif» (Jutant et al.,

31. http://www.club-innovation-culture.fr/grand-palais-nouvelle-application-mobile/, consulté le 26 septembre 2018.

32. Terme volontairement provocateur, utilisé par Rémi Susan (2011), http://www.internetactu.net/2011/03/01/les-ambiguites-de-la-gamification/, consulté le 20 novembre 2018.

33. Au sens strict, la quête relève d'une obstination personnelle à atteindre un objectif, elle ne suggère pas de mettre l'autre au défi, mais de s'y confronter, individuellement dans une dimension symbolique forte (la quête de Dieu, la quête du graal, la quête de la vérité). 
2009) qui aurait opéré une réécriture personnelle du dispositif d'exposition parce qu'il aurait utilisé les technologies associées à sa visite.

Selon la game-designeuse, Jane Mc Conigal (2011), la gamification du monde, via l'application des principes de jeux vidéo, est, ou sera, bénéfique à toutes choses, du jeu au coaching de soi, de la pédagogie aux serious game, etc. Si l'on suit cette idée, la gamification pourrait donc bénéficier également aux applications culturelles, or cela n'a été qu'esquissé. En effet, si les e-albums étaient réellement transmédiatiques, ils proposeraient de peindre de nouveaux tableaux d'Hopper, de dénicher des éléments dans l'œuvre qui ouvriraient sur de nouvelles perspectives (nouvelles scènes, nouvelles dérivations/évolution de l'univers) - c'est-à-dire seraient équivalents à ceux d'une "intrigue sans fin » ou la "narration augmentée », d'une œuvre de fiction (Jenkins, 2006). L'apprentissage ludique porté au pinacle par le discours d'accompagnement du serious game ne laissant pas l'institution indifférente. D'ailleurs, déjà habituée au développement des parcours de type «chasse aux trésors » pour son jeune public, la $R m n-G P$, envisageait de poursuivre dans cette voie et de mêler approche ludique et contenus culturels pour l'ensemble des publics ${ }^{34}$ (Appiotti, 2017). Cependant, le jeu n'est proposé qu'à un seul joueur car il s'agit de jeux solitaires qui entretiennent et proposent un nouveau rapport à l'œuvre dans une relation d'intimité sans pour autant que le défi y domine.

Pour Jenkins, le transmédia postule un monde particulier d'audience active et d'engagement des publics. Une œuvre transmédia proposerait donc des formes ouvertes de participation dans lesquelles les publics deviendraient co-créateurs du monde fictionnel $^{35}$. Au contraire, jusqu'alors les e-albums ne portaient que l'apparence de la nouveauté narrative, sans relever entièrement d'un monde fictionnel. Ils s'ancraient dans un plan d'exposition/démonstration d'une œuvre achevée, et non pas sur un exercice d'exploration/construction d'un univers en permanente, ou apparente expansion (simulacre d'évolution scénarisée, préencadrée). Rendre le public actif ! Voilà bien une des bases communes au transmédia storytelling et à la gamification. Cela ne se décrète pas, la valorisation du visiteur reste au niveau du discours institutionnel et d'une approche techniciste, puisque les potentialités du numérique pourraient transformer la médiation du musée en intégrant les logiques de marketing de l'expérience (Appiotti, 2017). L'injonction à la participation s'intègre également comme la forme de la gamification qui vise à capter l'attention de l'usager (Jahjah, 2014) pour alimenter les banques de données d'analyses

34. Voir également, L'atelier du midi, I'application ludique et participative de géolocalisation de I'exposition « Le Grand atelier du midi'. Cette application propose de se rendre sur le lieu du point de vue du peintre de photographier et de commenter sa prise de vue. https://www.grandpalais.fr/apps/mobile/appiphone.html, consulté le 27/11/2018.

35. http://www/transmedialab.org/autre/henry-jenkins-explique-sa-vision-du-transmedia-et-delengagement-des-publics/, consulté le 28/11/2018. 
statistiques du web collaboratif marchand et suggérer des contenus supplémentaires. En tant que professionnel du multimédia, Thomas Bijon ${ }^{36}$ met en doute la participation active du public qui selon lui se limite à liker et utiliser la géolocalisation ou la microgéolocalisation à l'intérieur même du parcours de l'exposition afin d'activer la fonction de reconnaissance des œuvres. Outre la catégorie des applications ludiques (Fabrique et Atelier, qui offrent de prendre des photos et de les transformer à la manière de...) qui sont basées sur une utilisation active de prises de vue, seuls les Mooc (Monet et Picasso ${ }^{37}$ ) ont impliqué le public en proposant une inscription gratuite pour suivre les chapitres sur la plateforme d'apprentissage à un rythme hebdomadaire. Le Mooc Picasso ${ }^{38}$, application mobile gratuite conserve l'essentiel des contenus du Mooc original ${ }^{39}$ la session « live » étant achevée depuis le 29 février 2015, les liens extérieurs à l'application de sont pas tous maintenus.

L'attrait économique n'était sans doute pas assez fort dans ces environnements numériques culturels ou à vocation d'information pour justifier le coût de développement d'un effet d'intrigue ou d'hypernarration (Lits, 2010). Il n'y a pas de storytelling dans ces e-albums : nous assistions en fait à une forme multimédia, certes très élaborée, mais une forme seulement d'évocation a posteriori. L'effet de découverte concernait sur les ealbums ce qui relève du passé, or l'effet de découverte qui concerne des œuvres de fiction transmédia en élaboration constante est celui de la promesse d'un futur, c'est-àdire un récit construit sur une intrigue ou une multiplicité de fils narratifs, mais surtout avec une quête et une récompense.

La quête d'abord, car ces applications préparaient à la visite en offrant le plan de l'exposition qui donnait au visiteur la possibilité de sélectionner en amont les œuvres qu'il souhaitait, et de gagner en efficacité une fois sur place en utilisant la microgéolocalisation. L'usager pouvait ainsi rationaliser sa visite. Il était libéré en quelque sorte de la scénographie générale de l'exposition et avait le choix de se construire un parcours de visite personnalisé. Pour autant, de la potentialité faisait-il acte ? La fonction de I'application muséale ou du e-album concourt à la réception de l'exposition, tout comme le catalogue d'exposition papier, mais le parallèle n'est pas complet. Si le catalogue assure une médiation en donnant les clés de lecture pour interpréter les œuvres et le cas échéant résoudre le problème posé par l'exposition, celle des e-albums qui offrent une visite virtuelle de l'exposition était bien mince car elle ne reprenait que quelques œuvres sur la totalité de l'exposition, les indices étaient donc partiels.

36. Interview Thomas Bijon par Françoise Paquienséguy.

37. https://culture.solerni.com/mooc/view.php?courseid=58, consulté le 24/11/2018.

38. https://www.grandpalais.fr/fr/article/mooc-picasso-inscrivez-vous-des-maintenant, consulté le $01 / 12 / 2018$.

39. Projet co-construit avec la fondation Orange (solerniculture) et la Rnm avec le soutien du Musée Picasso de Paris et le Centre Pompidou. 
La récompense, d'autre part: le Mooc Picasso proposait expressément comme récompense un badge à chaque niveau atteint et un super badge si on va jusqu'au bout des sept chapitres. Pour autant, la récompense symbolique accordée à l'usager fait bien partie du paradigme des techniques à la fois présentes dans le jeu et le marketing issus du courant uses and gratification (Bouquillion, Matthews, 2010). Mais ces exemples ne sont pas généralisables au domaine culturel et muséal, la quasi-totalité des Mooc quelle que soit la thématique proposent un système de badges, là encore la gamification reste discrète.

De fait, les e-albums n'exploitaient pas l'approche gamification. Ils ne semblaient pas intégrer à leur entier bénéfice les prouesses techniques ou scénaristiques qu'a apportées le jeu ${ }^{40}$ aujourd'hui. En somme, si nous assistions à une sorte de production gelée, figée dans le temps, à la façon des CD-Rom culturels, certes avec des arborescences plus adaptées puisque l'utilisateur avait, depuis, intégré une pratique hypermédiatique, (Nanard et Nanard, 1999), c'était tout simplement une question de sujet (le domaine culturel et artistique) et de forme d'un discours pédagogique, didactique ou informatif qui, lui, avait peu évolué. Nous notions qu'hormis de rares, et pas toujours convaincantes, démonstrations d'effets multimédias et interactifs sur certains sujets ${ }^{41}$, les expériences transmédia se bornaient, elles, à développer des œuvres de l'imaginaire de la culture mainstream ${ }^{42}$, plus aptes par ailleurs à capter un public massif venant justifier les considérables coûts de développement ou d'entretien des univers. Les e-album pourraient simplement revenir à leur fonction première, de simples galeries photo, de simples listes d'œuvres pour revenir à notre parallèle avec le catalogue papier (ce type d'application est d'ailleurs abondant sur smartphone et tablettes). Ils pourraient encore continuer à n'être que des équivalents plus ou moins homothétiques subordonnés à l'exposition tels le coffee table book posé sur la table basse... Ici, le e-album ne peut pas être mis en parallèle avec son homologue catalogue d'exposition car le support (tablette ou smartphone) de fait, le rend invisible au regard des autres. Ces environnements numériques pourraient rester si ce n'est déjà pas le cas un gadget numérique, produit dérivé de la boutique en ligne du musée.

Somme toute, s'il n'y a pas de trace de gamification massive dans les environnements numériques muséaux, il ne faut pas oublier qu'en matière d'usages et d'appropriation le sujet est et reste actif au regard des propositions des concepteurs et que le ludique a

40. Ce type de proposition avait été approché par un CD-Rom de la Rmn sur l'œuvre de Seurat Le cirque, Collection l'art en jouant 1996 CD-ROM, mais l'exemple n'a pratiquement pas été suivi.

41. Nous pourrions prendre ici comme exemple l'application jeunesse L'Ours, dans l'art préhistorique, qui propose un accès direct aux dix objets interactifs disséminés dans les trois chapitres de l'application, somme de représentations 3D de sculptures ou de calques qu'on actionne d'un doigt pour révéler les peintures rupestres.

42. Martel F. (2010). Mainstream. Enquête sur cette culture qui plaît à tout le monde, Flammarion, coll. « Essais », Paris. 
toujours sa nécessité et son importance «la consommation des nouvelles techniques s'opérationnalise autour de deux modèles d'usages dominants et a priori profondément opposés : le modèle rationnel et le modèle ludique » (Jouët, 1988). Rationnel et ludique participent à la structuration de nouveaux usages et sont facteurs d'appropriation des technologies car «la finalité prescrite de la technique comme instrument pratique et fonctionnel est détourné au profit d'usages ludiques » (Jouët, 1993). Cela étant posé, nous pourrions percevoir «la nouvelle application ${ }^{43}$, comme une nouvelle tentative à une dimension supérieure : le Grand Palais construit à travers elle un récit global et ouvert, alimenté par tous les récits clos de ses collections. La gamification serait enfin possible. L'univers serait celui du de la Réunion des Musées Nationaux que l'utilisateur alimente, occupe (dans lequel il vit), s'approprie, co-construit, auquel il se fidélise, dont il se réclame, cet univers fonctionne comme un métarécit. Il devient un transmédia storytelling global dont le coût lui-même est rationnalisé puisqu'une seule application s'offre au visiteur comme portail d'accès aux univers culturels.

\section{Conclusion : renouvellement et rationalisation}

La conclusion de cette réflexion conduit non pas au constat de l'innovation, mais à celui de la rationalisation des produits culturels, grandement liée à la numérisation des sources, quelles qu'elles soient (textes, tableaux, pages écran...) qui mène à une structuration très pensée et anticipée de l'environnement numérique des musées, et ce sera là notre ouverture finale. Une fois négociée, et les droits acquittés, la numérisation des documents liés aux expositions permet de diversifier les productions print, web ou numérique et de produire à chaque fois un produit culturel spécifique et ciblé. Ligne éditoriale qui court, nous l'avons vu, du catalogue d'exposition papier à l'application pour smartphone en passant par le site web dédié et se perpétue dans les visites virtuelles. Autrement dit, un storytelling pensé et rationnalisé se vérifie désormais pour chaque grande exposition ou événement, comme le projet patrimonial d'actualité du Nouveau Grand Palais ${ }^{44}$.

Mais la rationalisation œuvre également dans un autre registre : celui-là même de la conception des e-albums. En effet, ces derniers, comme les CD-Rom d'ailleurs, forment collections, à la $R m n-G P$, ou au Centre Georges Pompidou par exemple. Comme dans l'édition print, catalogues inclus, les collections de e-albums et applications ont des traits identifiants; on en compte quatre pour la Rmn-GP, si I'on en croit son site web, les

43. « L'application se veut ludique et permet d'enrichir ses photographies personnelles d'un choix d'icônes créées à partir des œuvres exposées [...] Il est possible de mettre en favori ses œuvres préférées d'un parcours, de prendre des notes, de faire une photo sans quitter l'application, d'associer ses propres prises de vues à l'ouuve favorite, de les partager par courriel ou sur les réseaux sociaux. » extrait du dossier de presse : http://presse.rmngp.fr/9577-2/, consulté le 23/11/2018.

44. https://www.grandpalais.fr/fr/article/decouvrez-le-nouveau-grand-palais, consulté le $19 / 12 / 2018$. 
recueils d'images, comme Les Fuvres des musées français, caractérisés par la haute définition des reproductions et les outils fournis pour l'exploiter et « leur inscription dans I'Histoire de l'Art» ; les applications d'expositions, comme Gauguin L'Alchimiste «pour apprendre au fil des expositions », caractérisées par une posture vulgarisatrice et pédagogique cherchant l'équilibre entre lire et voir ; les applications d'art qui ne valorisent pas un artiste mais un art, comme Jardins ou Joyaux par exemple, ou un sujet, comme Joséphine intime, et proposent donc une approche synthétique de celui-ci, façon Reader Digest et enfin la déclinaison des Fabriques et Ateliers qui proposent de faire, d'agir, de se prendre pour l'artiste et de tenter de l'imiter pour le comprendre, comme la Fabrique cubiste ou l'Atelier Picasso. Même si le résultat est assez décourageant en nombre de téléchargements, la logique de production en série de ces applications se poursuit et permet de réutiliser et de rentabiliser les coûts de développement, la leçon a été vite retenue par le Head of Digital and Multimedia du Grand Palais, Roei Amit « la Rnm-GP qui avait jusqu'alors l'habitude de développer une application par exposition (voir celles produites pour Gauguin l'alchimiste, Irving Penn ou encore Kupka) revoie sa stratégie afin de mieux répondre aux nouveaux usages des utilisateurs. En effet, des études ont montré que $41 \%$ des utilisateurs ne téléchargent plus aucune nouvelle application, tandis que cinq applications concentrent $85 \%$ du temps passé. Difficile alors de demander à ses visiteurs d'installer une nouvelle application à chaque exposition ${ }^{45}$. »

Facilité, et presque encouragé par le numérique, le déploiement organisé de l'environnement éditorial muséal contribue à l'industrialisation de la culture sur laquelle il est inutile de revenir : quel que soient l'artiste et son œuvre, le produit qui s'y consacrera le fera dans une forme donnée, voire normée par la collection. Comment alors dans ces conditions parler d'une innovation apportée par les applications et les e-albums ? Le constat n'y pousse pas, car en quoi ces applications culturelles actuelles - dès lors que ce ne sont pas de simples audio-guides - seraient-elles innovantes ? Il convient très nettement de relativiser, en oubliant le discours techniciste qui les promeut, et au contraire de se souvenir des caractéristiques des e-albums que nous avons posées. En synthèse, nous souhaiterions avancer qu'alors que les concepteurs et l'institution qui les emploie affichent une maîtrise des outils multimédias interactifs, ils ne font qu'employer les trouvailles des CD-Rom culturels et se heurtent toujours au défi esthétique que le catalogue papier devait déjà relever entre lire et voir, entre rationalité et expression artistique. De fait, ils reposent sur une systématisation des structures et des fonctionnalités ; ils vérifient une exploitation d'un fonds numérique commun, et s'intègrent à une stratégie de storytelling digne des plus grands groupes médiatiques

45. http://www.club-innovation-culture.fr/grand-palais-nouvelle-application-mobile/, consulté le $26 / 11 / 2018$. 
internationaux, même si nous remettons en question une partie de cette notion, comme nous l'avons vu.

En effet, malgré l'abondante production d'applications tactiles pour smartphones ou tablettes, celles-ci ne semblent être bien souvent que des avatars technologiquement plus élaborés des CD-Rom culturels tels qu'en développait la $R m n$ dans les années 1990. Si le multimédia se comprenait alors comme un recours à différents formats d'expression (textes, images, sons, vidéos), les applications proposées aujourd'hui ne font en fait que

bénéficier d'améliorations techniques développées grâce à l'essor considérable des techniques numériques et hypermédiatiques, mais ne convainquent pas pour autant en termes d'innovation de rhétorique numérique ou de construction narrative. Si changement il y a, on l'aura compris, c'est bien au niveau des stratégies de diffusion et de médiation des institutions muséales. Mais s'arrêter là serait oublier les aptitudes de l'usager et les progrès qu'il a faits (Paquienséguy et Collet, 2014; Saemmer, 2015) quant à la manipulation, à la géolocalisation, les réseaux sociaux et bien sûr la maîtrise des différents modes de consultation-lecture, bien que les applications développées ne semblent pas intégrer encore toutes les contributions de la gamification.

L'apport des environnements numériques que nous avons analysés serait sans doute à relier à une mutation de l'accès à la culture, à ses lieux, comme de sa consommation, autrement dit à la mutation des pratiques culturelles à propos des expositions des musées des beaux-arts. Elle reposerait à la fois sur les compétences de l'usager aujourd'hui très à l'aise avec l'interactivité, le multimédia et les objets connectés qui supportent les e-albums et sur un accès démultiplié à l'information (transmédia), dont la chronologie est totalement nouvelle (voir avant, revoir, voir pendant, lire ou regarder plusieurs choses en même temps), dont l'appropriation est quasi physique (toucher, zoomer, emporter avec soi, ...). La richesse des environnements numériques proposés, souvent gratuitement comme c'est le cas pour la nouvelle application du Grand Palais, représente à la fois une opportunité de démocratisation et de vulgarisation de l'Art, un accès facilité et approfondi à celui-ci via les thématiques des expositions, dont il ne reste qu'à se saisir à travers des technologies et fonctionnalités familières de notre vie quotidienne ; ce qui ne pouvait pas être le cas avec les lecteurs de CD-Rom et les ordinateurs des années 1990.

\section{Bibliographie}

Amar M., Vigué-Camus A. (2017). Lost in médiation ? Une étude d'usage des e-albums d'exposition. Livres d'art numériques, de la conception à la réception. Paris, Hermann, coll. « Cultures numériques », p. 63-96.

Appiotti S. (2017). La fabrique à créativité, De la co-conception à la modélisation des pratiques visuelles dans les applications muséales participatives. Livres d'art numériques, de la conception à la réception. Paris, Hermann, coll. «Cultures numériques », p. 203234. 
Bouquillion Ph., Matthews J. (2010). Le web collaboratif : mutations des industries de la culture et de la communication, PUG, Grenoble.

Chapelain B. (2011). De nouvelles médiations numériques au service de la culture augmentée. Revue Hermès, vol. 3, n 61, p. 106-108.

Collet L. (2018). Analyse de la performation dynamique des agences de communication : expérience utilisateur et formes multimédias interactives. Mémoire d'HDR en SIC, Université Lyon2.

Davallon J., Gottesdiener H., Le Marec J. (2000). Premiers Usages des cédéroms culturels, Ocim, Paris.

De Certeau M. (1980). L'invention du quotidien, tome 1. Arts de faire, Gallimard, coll. «Folio/Essais », Paris.

Durand A. (2009). Scénario multimédia. Formalisation moléculaire pour l'écriture de scénarii de documents multimédias. Document numérique, 2009/2 (vol. 12), p. 47-80.

Jahjah M. (2014). L'utilisation de la « gamification » dans la stratégie des acteurs du livre numérique: le cas Kobo. Mémoire du livre, Livre et jeu vidéo, vol. 5, $\mathrm{n}^{\circ}$ 2, http://id.erudit.org/iderudit/1024774ar

Jenkins H. (2006). Convergence Culture Where old and new media collide, NYU Press, Social Science, New York. Traduction (2013), La culture de la convergence. Des médias au transmédia, Armand Colin/Ina, Paris.

Jouët J. (1987). L'écran apprivoisé : télématique et informatique à domicile, CNET, Issyles-Moulineaux.

Jouët J. (1988). Nouvelles techniques, des formes de la production sociale. TIS, vol. $1, \mathrm{n}^{\circ}$ 3, p. 13-33.

Jouët J. (1993). Pratiques de communication et figures de la médiation. Réseaux, vol. 11, n60, p. 99-120.

Jutant C., Guyot A., Gentès A. (2009). Visiteur ou joueur ? Les multiples facettes de la technologie RFID. Lettre de I'OCIM, $\mathrm{n}^{\circ} 125$, p. 12-20.

Laborderie A., Juhel F. (2016) Les relations texte/image dans l'édition numérique enrichie d'une œuvre littéraire : Le cas de l'application Candide de la BnF. Itinéraires. Littérature, textes, cultures, Pléiade (EA 7338), Ethos numérique.

Lavigne M. (2010). Multimédia culturel, l'impossible industrie. Cultures, technologies et mondialisation, Paris, L'Harmattan, p. 79-86.

Lavigne M. (2007). Regard rétrospectif sur les CD-rom Culturels. rapport de recherche, 2017 https://www.academia.edu/10989401/Regard_rétrospectif_sur_les_CD-

ROM_culturels

Le Diberder A. (2006). Histoire du multimédia : un succès et deux enterrements. Hermès $\mathrm{n}^{\circ} 44$, p. 33-39.

Le Marec J. (2004). Systèmes interactifs et CD Rom de musées. Chaudiron, Stéphane (dir.) Evaluation des systèmes de traitement de l'information, Hermès science publication. 
Lits M. (2010). Du Récit au récit médiatique. De Boeck, $2^{\mathrm{e}}$ édition, première édition 2008, Bruxelles.

Mayon M. (2016). Le catalogue d'exposition est-il en pleine mutation ? Le Monde du Livre, https://mondedulivre.hypotheses.org/5314

Mc Conigal J. (2011). Reality is broken: why game make us better et how they can change the world, NYC, Penguin Books, New York.

Nanard M., Nanard J. (1999). Documents hypermédias : Définitions et concepts associés, Techniques de l'ingénieur Représentation et traitement des documents numériques, H7208, Éditions T.I., https://www.techniques-ingenieur.fr/base-documentaire/ technologiesde-I-information-th9/representation-et-traitement-des-documents-numeriques-

42312210/documents-hypermedias-h7208/

Odin R. (2000). De la fiction, De Boeck Université, coll. « Arts et cinéma », Bruxelles.

Ottinger D. (2012). Edward Hopper : Ombre et lumière du mythe américain, Découvertes Gallimard - Rmn-GP, Paris.

Paquienséguy F., Collet L. (2014). Les produits interactifs multimédias réinventés ? Des cd-rom culturels aux e-albums des tablettes. Livre-post numérique : histoire, mutations et perspectives. Paris, Europia, p. 1-10.

Paquienséguy F. (2018). Multimédias interactifs, les progrès de l'usager. Théorème, $\mathrm{n}^{\circ} 29$, p. 97-108.

Saemmer A. (2015). Rhétorique du texte numérique. Figures de la lecture, anticipations de pratiques, ENSSIB, coll. « Papiers », Villeurbanne.

Schafer V., Thierry B. (2011). Le mariage de raison du musée d'art et du Web, Hermès, La Revue, 2011/3 (n० 61), p. 102-105.

Séguy F. (1995). Le multimédia du foisonnement à la raison. Les Cahiers du spectacle et de l'audiovisuel, n6, p. 79-85.

Séguy F. (1999). Les Produits interactifs et multimédias : méthodologie, conception, écriture, PUG, Grenoble.

Vidal G. (2006). Contribution à l'étude de l'interactivité les usages du multimédia de musée. Presses universitaires de Bordeaux, coll. Labyrinthes, Bordeaux. 